\title{
Monte Carlo calculations of the REBUS critical experiment for validation of burn-up credit
}

\author{
M. Hennebach and H. Kühl \\ WTI GmbH, 52428 Jülich, Germany
}

\begin{abstract}
The REBUS experiment is a valuable benchmark for the validation of Monte Carlo criticality codes within the context of burn-up credit. It investigates the difference in reactivity worth of unirradiated fuel rods and of fuel rods irradiated in a PWR. This paper presents results of criticality calculations for this experiment with the Monte Carlo codes MCNP and SCALE/KENO. Furthermore, calculated results for the 3D core characteristics (fission rate and flux distribution) are compared with the measured values.
\end{abstract}

\section{Introduction}

The application of burn-up credit (BUC) to criticality safety analysis for spent nuclear fuel (SNF) configurations requires the implementation of both estimation of the SNF isotopic composition with the aid of depletion calculation tools and estimation of the SNF reactivity with the aid of criticality calculation tools.

While depletion calculation tools have been developed and validated over many years, the calculation tools to predict the SNF reactivity outside the core in storage, transport or disposal conditions are not validated as thoroughly. Since out-of-core SNF operations are usually made without any reactivity monitoring devices, more reliance is placed on the accuracy of the calculated neutron multiplication factor. For these reasons, there is a strong requirement for validation of BUC criticality safety applications.

\section{The REBUS experiment}

The REBUS experimental program [1], distinguished by a combination of chemical analysis and critical experiment, provides a valuable benchmark for this validation effort.

It investigates the difference in the reactivity worth of unirradiated and irradiated fuel rods. In the paper on hand, the results attained for samples irradiated to a burn-up of approx. $54 \mathrm{GWd} / \mathrm{t}_{\mathrm{HM}}$ in a pressurized water reactor (PWR) are evaluated. These samples are cut from the middle of the PWR rods to ensure a flat axial burn-up profile (see fig. 1).

Since whole bundles of fuel rods are used, the change in reactivity is significant enough to be observable by Monte Carlo methods. This is a feature of prime importance in the REBUS program, allowing a more direct validation of the calculation code schemes commonly used in criticality safety analysis (i.e., estimation of $\mathrm{k}_{\mathrm{eff}}$ rather than reactivity perturbation calculations).

The REBUS bundles consist of 25 fuel rods and are placed in a $27 \times 27$ driver zone of VENUS fuel rods surrounded by a water reflector, cf. ref. [1]. The water level of the VENUS critical facility is used to control the neutron multiplication

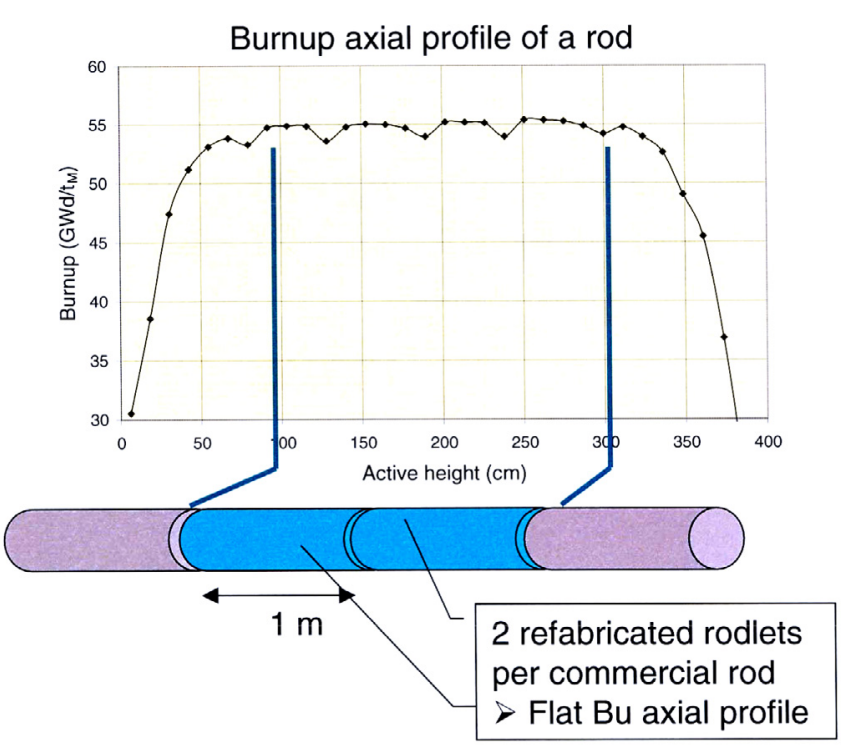

Fig. 1. Fabrication of the irradiated REBUS fuel rods.

of the setup. In addition to measurements of the reactivity effects and critical heights of the water level for the fresh and the irradiated fuel bundles, axial and horizontal fission rate distributions are determined by gamma scans, and horizontal neutron flux distributions are monitored with the aid of activation sensors (scandium wires). Furthermore, a detailed chemical assay of one irradiated fuel sample is performed. The analyzed nuclides were selected on the basis of their reactivity importance or for their applicability as burn-up indicators. Nuclide densities for 18 major and minor actinides, 19 fission products (the most important neutron absorbers) and 4 burnup indicators $\left({ }^{148} \mathrm{Nd},{ }^{150} \mathrm{Nd},{ }^{144} \mathrm{Ce}\right.$ and $\left.{ }^{137} \mathrm{Cs}\right)$ are provided by the radiochemical analysis, along with several other isotopes of interest.

The experimental results obtained for fresh and irradiated fuel are applied herein to directly benchmark the criticality codes KENOV of the SCALE-5 system and MCNP5. 


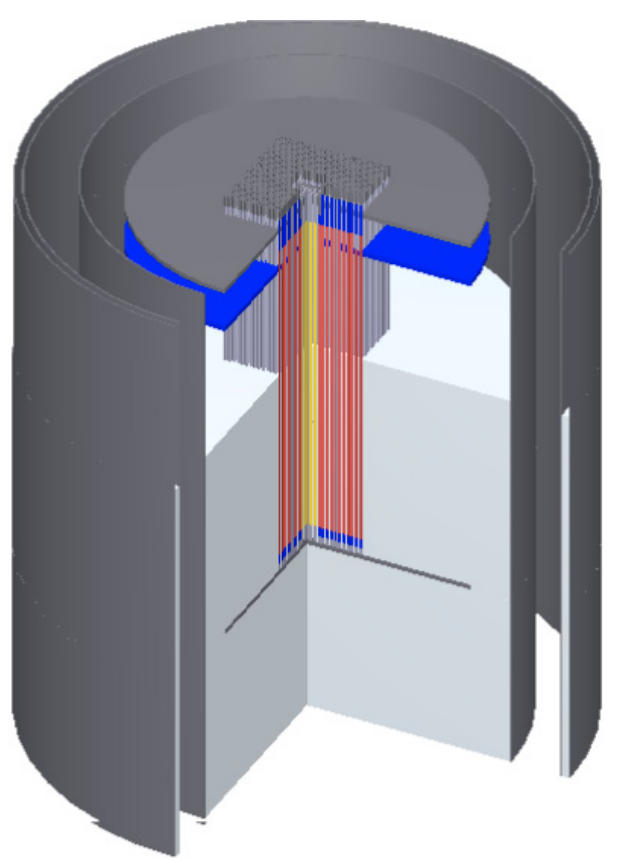

Fig. 2. 3D view of the VENUS/REBUS calculation model.

\section{Criticality calculations}

Monte Carlo calculations for the REBUS setup with fresh and with irradiated fuel can provide answers on several aspects of BUC criticality safety applications. The important experimental result here is the change in reactivity worth between the fresh and the irradiated fuel bundle, expressed as the difference in the critical water level for both setups.

Using the chemical assay data for the irradiated fuel rods, a comparison of the calculated and the measured reactivity worth shows how well the criticality codes can model the influence of the variations in fuel composition due to irradiation, i.e., the reactivity loss due to decreasing net fissile content on the one hand and build-up of strong neutron absorbers on the other hand.

As noted in the introduction above, our criticality analysis of the REBUS experiment is performed with the Monte Carlo simulation codes MCNP5 and KENO V (part of the SCALE5 system), both of which see widespread use in criticality safety applications. The geometry of the VENUS reactor with the REBUS bundle at its core is represented in detail in the calculation model (see figs. 2 and 3).

Regarding neutron cross section data, MCNP5 with pointwise continuous cross section data based on ENDF/B-V and ENDF/B-VI data is used, whereas the codes of the SCALE-5 system include the use of 238-group and 44-group ENDF/BV/VI derived cross section libraries. The 44-group library, collapsed from the 238-group library, has been especially developed for the analysis of well moderated fresh and spent light water reactor fuel configurations.

The crucial experimental value representing the reactivity loss of the fuel rods during irradiation is the difference in critical water level for the REBUS setups with fresh and with irradiated fuel. To calculate this value, $\mathrm{k}_{\mathrm{eff}}$ is determined as a function of the water level to derive the critical water level

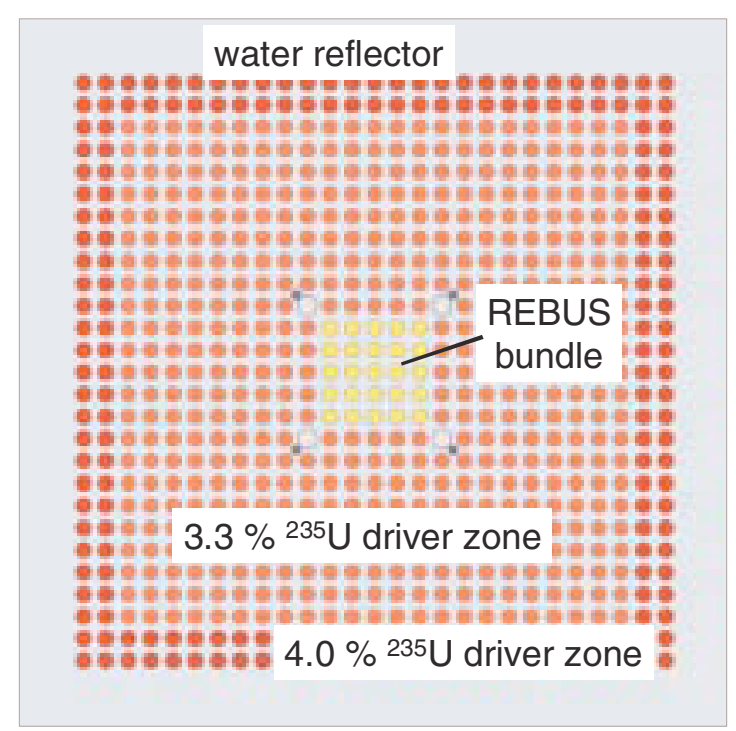

Fig. 3. Horizontal cut of the VENUS/REBUS core.
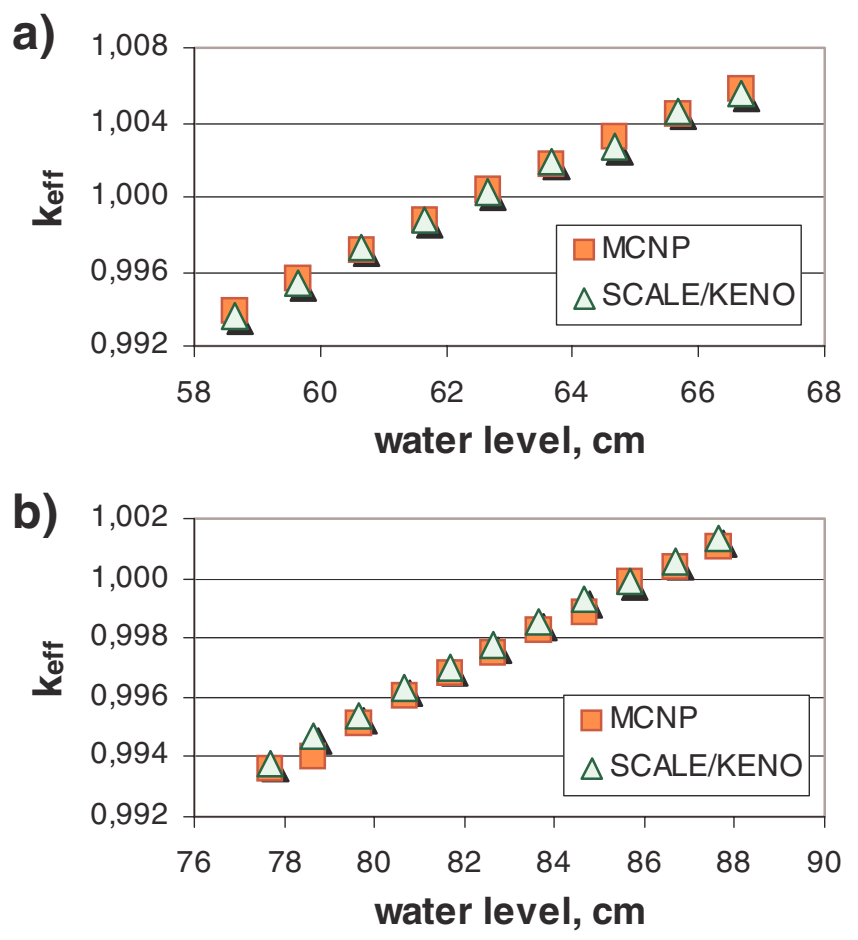

Fig. 4. Dependence of $k_{\text {eff }}$ on water level $h$ for a) the unirradiated, and $b$ ) the irradiated REBUS bundle.

$\mathrm{h}_{\mathrm{C}}\left(\right.$ with $\left.\mathrm{k}_{\mathrm{eff}}=1\right)$ by linear approximation. Since the reactivity effect of the water level $\Delta \mathrm{k}_{\text {eff }} / \Delta \mathrm{h}$ ) is part of the measurement program, this also provides a good point of comparison between experiment and Monte Carlo simulation.

Figure 4 shows the criticality calculation results for fresh and for irradiated fuel respectively, with the fuel composition for the latter given by the chemical assay. The statistical uncertainties of the Monte Carlo calculations are about as large as the data point size.

The steady inclination of the data points shows that the derivation of the critical water level by linear approximation is 
a reliable calculation model. Results for both REBUS bundles are given in tables 1 and 2 below.

Table 1. Critical water level $h_{C}$ and reactivity effect $\Delta \mathrm{k}_{\text {eff }} / \Delta \mathrm{h}$ for the unirradiated REBUS bundle.

\begin{tabular}{ccc}
\hline unirradiated & $\mathrm{h}_{\mathrm{C}}, \mathrm{cm}$ & $\Delta \mathrm{k}_{\mathrm{eff}} / \Delta \mathrm{h}, \mathrm{pcm} / \mathrm{cm}$ \\
\hline experiment & $59.05 \pm 0.03$ & $153 \pm 2$ \\
MCNP & $62.548 \pm 0.002$ & $148 \pm 2$ \\
SCALE & $62.649 \pm 0.002$ & $150 \pm 2$ \\
\hline
\end{tabular}

Table 2. Critical water level $\mathrm{h}_{\mathrm{C}}$ and reactivity effect $\Delta \mathrm{k}_{\text {eff }} / \Delta \mathrm{h}$ for the irradiated REBUS bundle.

\begin{tabular}{ccc}
\hline irradiated & $\mathrm{h}_{\mathrm{C}}, \mathrm{cm}$ & $\Delta \mathrm{k}_{\mathrm{eff}} / \Delta \mathrm{h}, \mathrm{pcm} / \mathrm{cm}$ \\
\hline experiment & $81.38 \pm 0.04$ & $74.6 \pm 0.5$ \\
MCNP & $86.037 \pm 0.002$ & $76.2 \pm 1.4$ \\
SCALE & $85.781 \pm 0.002$ & $74.4 \pm 1.5$ \\
\hline
\end{tabular}

While the calculated reactivity effect $\Delta \mathrm{k}_{\mathrm{eff}} / \Delta \mathrm{h}$ agrees very well with the measured value for both the irradiated and the unirradiated bundle, the critical water levels $\mathrm{h}_{\mathrm{C}}$ are not represented as accurately, with overestimations of up to $5 \mathrm{~cm}$. This overestimation corresponds to an under-estimation of the measured $\mathrm{k}_{\mathrm{eff}}$ of approximately $500 \mathrm{pcm}$, which is not atypical for criticality benchmark experiments, but definitely larger than the statistical uncertainties would allow for.

Although the calculations were made for nominal dimensions and material specifications of the experimental setup, the possible effects of tolerances in geometry and material compositions were investigated and can be excluded as possible sources of this deviation.

The difference in critical water levels $\Delta \mathrm{h}$ due to the reactivity loss under irradiation is determined for both criticality codes. The measured and calculated results for $\Delta \mathrm{h}$ are given in table 3 below.

Table 3. Difference $\Delta \mathrm{h}$ in critical water level for unirradiated and irradiated fuel, $\mathrm{cm}$.

\begin{tabular}{lll}
\hline experiment & MCNP & SCALE \\
\hline $22.33 \pm 0.04$ & $23.489 \pm 0.003$ & $23.132 \pm 0.003$
\end{tabular}

Results for both criticality codes lie within $1.2 \mathrm{~cm}$ of the measurement, which means that the reactivity difference due to irradiation can be predicted within a margin of $6 \%$. This is remarkable in the light of the fact that this reactivity loss is comparably small with an equivalent $\Delta \mathrm{k}$ of approx. $2000 \mathrm{pcm}$.

\section{3D core characteristics}

The usefulness of the criticality calculation results presented above is, of course, highly dependent on the quality of the calculation model. To ensure that the level of detail of the model is appropriate, calculated radial distributions of flux and fission rate are compared with the results from measurements

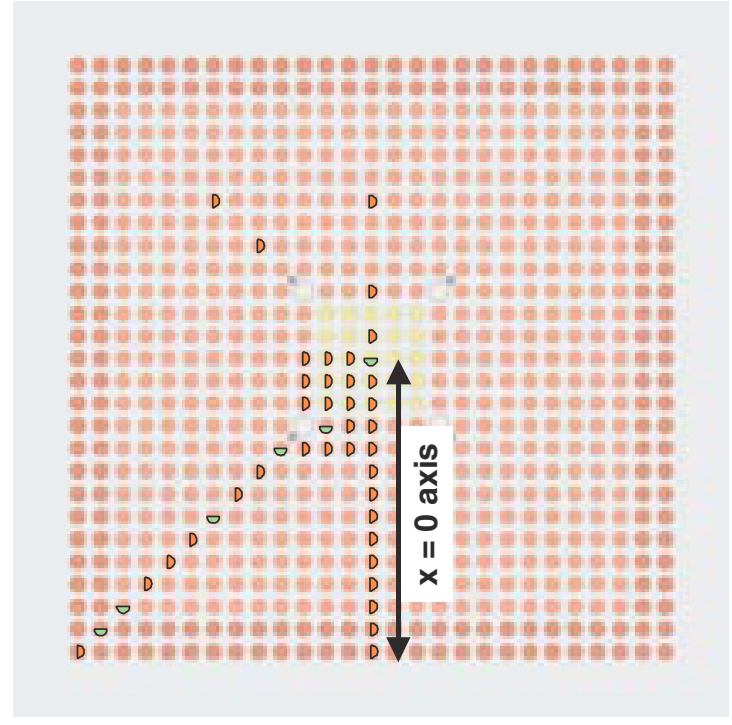

\section{$D$ fission rate measurement \\ $\checkmark$ axial fission rate measurement}

Fig. 5. Positions of fission rate masurements.
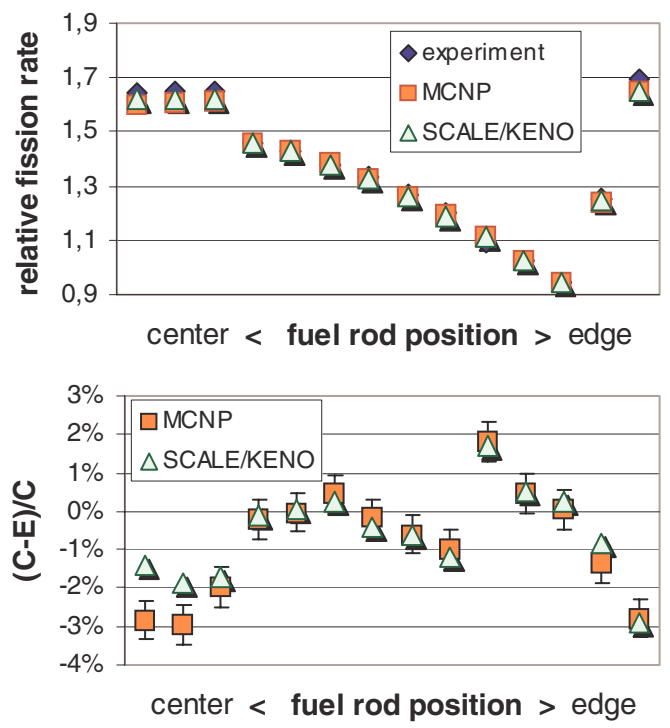

Fig. 6. Fission rate distribution along the $\mathrm{x}=0$ axis.

for the unirradiated REBUS bundle - if the neutron distribution in the core is not well represented by the calculation, one cannot expect to gain good results for $\mathrm{k}_{\mathrm{eff}}$.

Positions with measured fission rates for the VENUS core with the unirradiated REBUS bundle are shown in figure 5.

Results for the calculated and the measured radial fission rate distribution along the $\mathrm{x}=0$ axis are plotted in figure 6 .

The core behaviour is very well represented by calculations, with only a few percent deviation between measured and calculated results. The increase at the edge of the REBUS core is due to the higher enrichment of the outer two rows of the driver zone (see fig. 3). Results for the distribution along the diagonal axis, which are not shown here, are of a similar quality. 

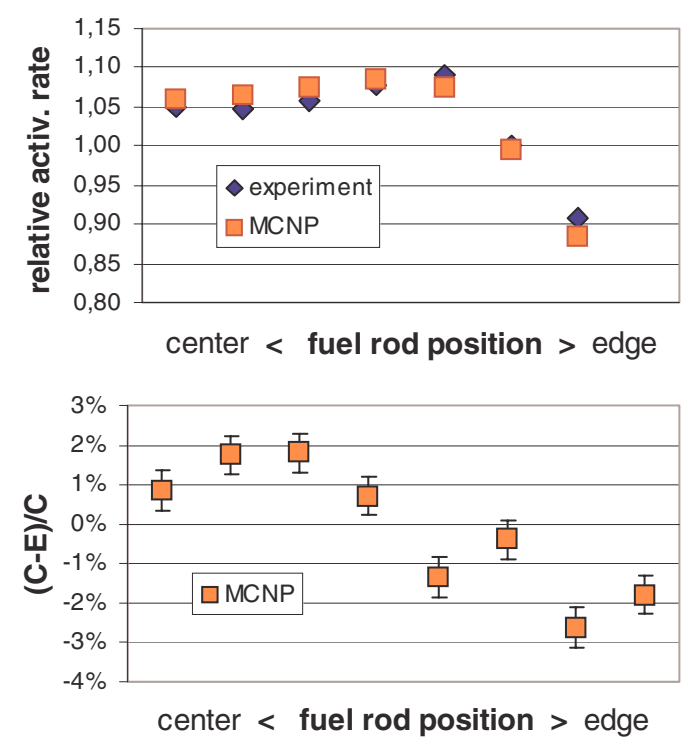

Fig. 7. Sc activation rate distribution along the $\mathrm{x}=0$ axis.

It is especially noteworthy that the agreement between the Monte Carlo codes SCALE/KENO and MCNP is even better, in spite of different treatments of the neutron cross sections.

Calculated results for the relative scandium activation rates (reflecting the flux distribution in the core) along the $\mathrm{x}=0$ axis, shown in figure 7, also agree well with the measured values. Activation rates are calculated with MCNP only, since SCALE/KENO does not provide an easy transition from calculated fluxes to detector responses (i.e., activation rates).

\subsection{Core calculations for the VENUS-2 MOX benchmark}

An MCNP calculation of the fission rate and flux distributions for the NEA VENUS-2 MOX benchmark [2] shows results of similarly good quality. In this experiment, equivalent fission fluxes for a mixed $\mathrm{UO}_{2} / \mathrm{MOX}$-core were measured using ${ }^{58} \mathrm{Ni},{ }^{115} \mathrm{In},{ }^{103} \mathrm{Rh},{ }^{64} \mathrm{Zn},{ }^{237} \mathrm{~Np}$ and ${ }^{27} \mathrm{Al}$ detectors at several important positions in the reactor.

A cross section of the MCNP calculation model of a quarter core is shown in figure 8 . The results for the flux detector rates and for axial and radial fission rate distribution, calculated with MCNP-4C2 and neutron cross sections based mostly on ENDF/B-VI data, agree very well with the measured values and compare favourably with the results for other calculation codes (cf. ref. [2]).

\section{Conclusions}

The paramount feature of the REBUS program is to provide a direct benchmark for the reactivity loss of $\mathrm{UO}_{2}$ fuel during

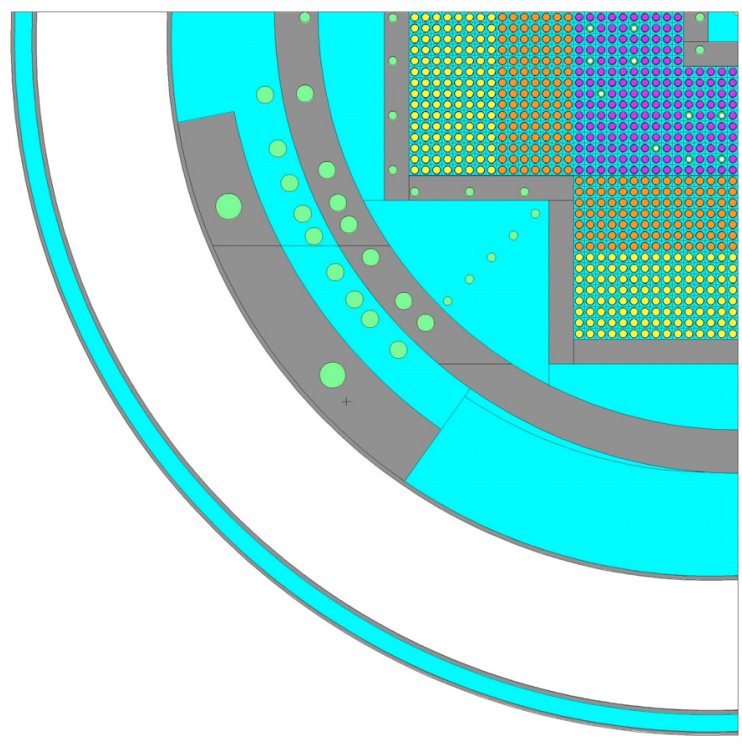

Fig. 8. MCNP calculation model of the VENUS-2 MOX quarter core. Detector positions are marked as light green circles.

irradiation in a commercial PWR, and the results given herein show that even the comparatively small reactivity loss of approximately $2000 \mathrm{pcm}$ measured in this experiment can be calculated within an accuracy of $6 \%$. The validity of this main result is strengthened by an overall good representation of the measurement.

A detailed comparison of measured and calculated REBUS core characteristics (fission rate and flux distributions) shows good agreement, which indicates an adequately detailed model geometry for the criticality calculations. The slight underestimation of absolute $\mathrm{k}_{\mathrm{eff}}$ values is not out of the typical range for criticality benchmark experiments but is, nevertheless, still under scrutiny.

All in all, our evaluation of the REBUS experiment with PWR fuel validates the usability of Monte Carlo criticality codes in BUC applications.

Results for combined depletion and criticality calculations, performed with several commonly used depletion calculation codes, are presented in [3].

\section{References}

1. P. Baeten et al., in Proceedings of the International Conference on Nuclear Criticality Safety ICNC 2003, Tokai Mura, Japan, Oct. 2003, pp. $645-649$.

2. VENUS-2 MOX-fuelled Reactor Dosimetry Calculations, Final Report, OECD/NEA Report, NEA/NSC/DOC(2005), ISBN 9264-01084-X (2006).

3. F. Attale et al., in Proceedings of the International Conference on Nuclear Criticality Safety ICNC 2007, St. Petersburg, Russia, May 2007 (to be published). 